\title{
A review of thiazide-induced hyponatraemia
}

\author{
Emmanuel Eroume A Egom, Debora Chirico and Andrew L Clark
}

\begin{abstract}
There are numerous reports of thiazide-induced hyponatraemia (TIH) and its incidence is growing as a result of increasing prescription after guidelines recommending thiazides as first-line treatment of essential hypertension have been introduced. Thiazide diuretics are a common cause of severe hyponatraemia that is usually induced within two weeks of starting the thiazide diuretic, but it can occur any time and very rapidly in susceptible patients. Despite several relevant reports and years of clinical experience, TIH remains a very common clinical scenario. Although its impact has recently been reviewed, little attention has been given to the practical approach for preventing this medical complication. In the present review, the epidemiology, pathogenesis, clinical presentation and management of TIH are discussed, and an approach to its prevention suggested in the hope that increased awareness and understanding will reduce the incidence and complications of this potentially life-threatening condition.
\end{abstract}

KEY WORDS: hyponatraemia, thiazide, thiazide-induced hyponatraemia

\section{Introduction}

Thiazide diuretics have a primary action of inhibition of sodium and chloride reabsorption in the distal convoluted tubule of the nephron. They have been used for the treatment of hypertension since their discovery in $1957 .{ }^{1}$ They reduce blood pressure when administered as monotherapy, enhance the efficacy of other antihypertensive agents, and decrease hypertension-related morbidity and mortality. ${ }^{2}$ However, their use is limited by electrolyte and metabolic disturbances, such as hyponatraemia, hypokalemia and insulin resistance. ${ }^{3}$ Since the first detailed description of thiazideinduced hyponatraemia (TIH) over four decades ago, numerous additional cases have been reported. ${ }^{4}$ Hyponatraemia is common with thiazide use, and has an estimated incidence of $11 \%$ in one series of 114 elderly patients. ${ }^{5}$ The impact of TIH has recently been reviewed, but little attention has been given to a practical approach to help prevent it. ${ }^{6}$ In this review, the epidemiology, pathogenesis,

Emmanuel Eroume A Egom, ${ }^{1,2}$ academic clinical fellow in cardiology and metabolic medicine; Debora Chirico, ${ }^{1}$ foundation trainee; Andrew L Clark, ${ }^{1,2}$ chair in clinical cardiology and honorary consultant cardiologist

\footnotetext{
${ }^{1}$ Hull and East Yorkshire Hospitals NHS Trust, Castle Hill Hospital, Cottingham, Yorkshire

2 Division of Cardiovascular and Respiratory Studies, Hull York Medical School/University of Hull, Castle Hill Hospital, Cottingham, Yorkshire
}

clinical presentation and management of TIH will be discussed and an approach to its prevention suggested in the hope that increased awareness and understanding will reduce the incidence and complications of this potentially life-threatening condition.

\section{Epidemiology}

The major risk factors for TIH are old age, female sex, lower body weight, hypokalaemia and concurrent use of other medications that impair free water excretion. ${ }^{7-9}$ The elderly appear to be more prone to TIH as they do not excrete free water as efficiently as younger subjects. ${ }^{9}$ Blunted prostaglandin synthesis may contribute, along with polypharmacy, particularly including nonsteroidal anti-inflammatory agents. Hypertensive women are particularly at risk of hyponatraemia. ${ }^{6-10}$ Women may drink more than men to compensate for the diuretic and therefore develop more dilutional hyponatraemia, and hypertensive women may be more often treated with diuretics than hypertensive men. There is no clear published evidence why women may be more likely to drink excessively than men. However, in general, women have less fat and muscle than men and so have a smaller store of body fluids predisposing them to become dehydrated more easily. Furthermore, dietary patterns may have a role. There is an emphasis in the popular literature on diet and 'healthy living' that maintaining a high fluid intake is important (despite the lack of any evidence to support such a statement). At any given time half of the adult women and nearly a third of the adult men in the USA are dieting. ${ }^{11}$ Therefore many women may be drinking larger volumes of fluid per kilogram of dry weight than men. An additional factor is that many women have been encouraged to have a high fluid intake to decrease the risk of urinary tract infection. The evidence that women might be more likely to be prescribed thiazide than men came from a study conducted in the early 1970s in Sweden where, in a health screen, 1,034 of 15,903 persons between 20 and 63 years of age who were taking thiazides, $66 \%$ were women. ${ }^{12}$ Furthermore, in a population-based descriptive study carried out by LaCroix and colleagues to look at the effect of thiazide diuretic agents on the incidence of hip fracture, trained interviewers conducted household surveys to collect information on the use of prescription and nonprescription drugs among 9,518 men and women 65 years of age or older and showed that among the users of thiazides, $71.6 \%$ were women. ${ }^{13}$ It is also true that comorbidities (eg co-existence of benign prostatic hypertrophy) or side effects (eg women suffer more from coughing induced by angiotensin-converting enzyme inhibitors), of which the prevalence may differ between men and women, may cause sex differences in the choice of antihypertensive drugs. 
Women may also be more symptomatic than men at comparable serum sodium levels and therefore may be diagnosed more often than men. The reasons why women are more prone to symptoms at similar sodium concentrations than men remain unclear. Women may be more sensitive to the effect of hyponatraemia than men: when hyponatraemic encephalopathy develops, for example, women are approximately 25 times more likely to die or have permanent brain damage than men. ${ }^{14}$ At any given body weight, women have less fluid than men (and more fat). Hence a $70 \mathrm{~kg}$ man with a sodium of $132 \mathrm{mmol}^{-1}$ has much more sodium on board than a $70 \mathrm{~kg}$ woman and a sodium of $132 \mathrm{mmol} . \mathrm{l}^{-1}$. Plasma sodium level is determined by the ratio between the amount of exchangeable sodium and potassium and total body water: a small change in total body sodium will have a more profound effect on serum sodium in a person with less total body water. ${ }^{10}$ Furthermore, small body mass or muscle wasting may also imply underlying disease. ${ }^{15}$ Hypokalaemia is an independent predictor of $\mathrm{TIH}$, supporting the concept of sodium-potassium movement proposed almost half a century ago. ${ }^{8,16}$ In the presence of a large potassium deficit, transcellular ion exchange (potassium exits and sodium enters cells) might contribute to hyponatraemia.

\section{Pathogenesis}

TIH occurs when the intake of free water is greater than the amount the kidneys are able to excrete. In subjects with a moderate impairment of free-water excretion, a thiazide will cause hyponatraemia if fluid intake is excessive. Hyponatraemia can even happen in those with more serious impairment of free-water excretion with ordinary fluid intake. In patients who are hypovolemic, anti-diuretic hormone $(\mathrm{ADH})$ is stimulated and contributes to reduced freewater excretion. In contrast, in euvolemic or hypervolemic patients, $\mathrm{ADH}$ is usually suppressed and is not contributory. ${ }^{3}$

Thiazides block sodium chloride co-transport in the distal convoluted tubule. As a result, sodium excretion is increased, while excretion of free water is reduced. ${ }^{6,9}$ Several drugs increase the likelihood of developing TIH. Non-steroidal anti-inflammatory drugs, through prostaglandin inhibition, decrease freewater clearance. ${ }^{9}$ Drugs such as chlorpropamide or selective serotonin reuptake inhibitors may be associated with inappropriate $\mathrm{ADH}$ secretion, which predisposes to TIH. Other drugs commonly associated with an increase risk of hyponatraemia are summarised in Table 1. Polydipsia caused by psychotropic medications also predisposes to TIH. ${ }^{3}$

Table 1. Drugs commonly associated with hyponatraemia.

\begin{tabular}{|c|c|c|}
\hline Class & Drug & Mechanism \\
\hline \multirow[t]{3}{*}{ Diuretics (thiazide and loop) } & Indapamide & Decreases total body sodium \\
\hline & Chlorothiazide & \\
\hline & Frusemide & \\
\hline \multirow[t]{6}{*}{ Antidepressants } & Sertraline & SIADH production \\
\hline & Fluoxetine & \\
\hline & Paroxetine & \\
\hline & Citalopram & \\
\hline & Venlafaxine & \\
\hline & Modobemide & \\
\hline Anticonvulsants & Carbamazepine & $\begin{array}{l}\text { SIADH production and increase the sensitivity } \\
\text { of the distal tubule to ADH }\end{array}$ \\
\hline \multirow[t]{2}{*}{ ACE inhibitors } & Enalapril & SIADH production? \\
\hline & Captopril & \\
\hline NSAIDs & Celecoxib & Increase the sensitivity of the distal tubule to ADH? \\
\hline \multirow[t]{2}{*}{ Hormone analogues } & Desmopressin & Exogenous antidiuretic hormone \\
\hline & Oxytocin & \\
\hline \multirow[t]{5}{*}{ Chemotherapeutics } & Vincristine & SIADH production \\
\hline & Vinblastine & \\
\hline & Carboplatin & \\
\hline & Cisplatin & \\
\hline & Cyclophosphamide & \\
\hline Hypnotic & Temazepam & SIADH production \\
\hline Antipsychotic & Dozapine & SIADH production \\
\hline
\end{tabular}

$\mathrm{ACE}=$ angiotensin-converting enzyme; $\mathrm{ADH}=$ anti-diuretic hormone; NSAID = non-steroidal anti-inflammatory drugs; $\mathrm{SIADH}=$ syndrome of inappropriate antidiuretic hormone hypersecretion. 


\section{Clinical presentation}

The clinical manifestations of TIH are similar to those due to other causes of hyponatraemia and vary from mild fatigue or nausea to severe neurologic compromise, including death. ${ }^{7} \mathrm{TIH}$ may be acute or gradual in onset and ranges from mild to severe and from asymptomatic to symptomatic. One of the most remarkable features of the condition is the rapidity with which it can occur. The onset is within two weeks of starting the diuretic in $50-90 \%$ of cases, but it can occur within a day or two or even after a single dose when there are complicating features such as a reduction of renal function with aging, changes in water or sodium intake, and concurrent use of other medications that impair free-water excretion. ${ }^{7}$ Mild hyponatraemia, with serum sodium concentration in the range of 125-132 mmol. $\mathrm{l}^{-1}$, is usually asymptomatic, although vague symptoms such as fatigue or nausea are possible. ${ }^{3}$ More severe hyponatraemia can be asymptomatic or associated with symptoms including weakness, vomiting, nausea, confusion, dizziness, abdominal pain, lethargy, seizures and even coma. ${ }^{17}$

\section{Management}

The initial key step in the approach and evaluation of TIH is the awareness that it can happen. The acute management is determined more by the presence of neurological symptoms than by the sodium level per se. Patients with acute onset of hyponatraemia (decrease in sodium concentration to less than $120 \mathrm{mmol} . \mathrm{l}^{-1}$ in less than 48 hours) are more likely to be symptomatic and are more at risk of cerebral oedema than those in whom hyponatraemia developed more gradually. ${ }^{18}$ The vast majority of patients do respond only to drug withdrawal and fluid restriction.

In patients with hypovolemic $\mathrm{TIH}$, who have raised $\mathrm{ADH}$ contributing to reduced free-water clearance, isotonic saline infusion is appropriate because it will restore volume and suppress $\mathrm{ADH}$ release. As it is also true that normal saline can exacerbate hyponatraemia in patients with syndrome of inappropriate antidiuretic hormone hypersecretion (SIADH), who may excrete the sodium and retain the water, decisions about management should consider ongoing renal free water and solute losses. The combination of normal saline and a loop diuretic can also elevate the serum sodium concentration. This latter approach often is useful for patients with high urine osmolality, because the loop diuretic acts to reduce urine osmolality. In patients with severe and serious manifestations, such as seizures or coma, hypertonic saline is recommended. ${ }^{3}$ In patients with euvolemia or hypervolemia-associated $\mathrm{TIH}$, who tend to have lower serum levels of urea, and creatinine, normal saline will not restore free-water clearance or serum sodium concentration. Hypertonic saline will predictably raise serum sodium concentration. Furosemide in addition to the hypertonic saline infusion can increase free-water clearance and thus hasten the increase of the serum sodium concentration while avoiding volume overload: such an approach may be of great value especially in the presence of oedema-producing states. ${ }^{19}$ In general, hypertonic saline should only be used rarely for severely symptomatic patients thought to be in grave danger. Because of the risk of central pontine myelinolysis, hypertonic saline should only be given by experienced teams with close supervision.

In patients with mild or no symptoms, counselling about the risks and benefits of the continued use of the thiazide diuretic, avoidance of excessive water intake, and careful monitoring of the serum sodium level is usually all that is needed. Normal saline is not needed unless correction of volume depletion is needed. Increasing oral salt intake in combination with fluid restriction can increase serum sodium concentration. ${ }^{3}$

\section{Prevention}

The first step in prevention is awareness that TIH can happen, particularly in elderly women, small individuals, individuals with a high fluid intake and possibly those whose usual serum sodium or potassium concentration is in the low-normal range.

Since most cases of TIH develop within one to two weeks, electrolytes should be measured within two weeks of starting treatment. $^{3}$ If the serum sodium level falls more than a few mmol. $1^{-1}$ below the bottom end of the normal range, the diuretic should be stopped. However, in susceptible individuals, the serum sodium may fall in hours of diuretic administration, and severe hyponatraemia can develop within two days. ${ }^{7}$ Therefore in susceptible patients, serum sodium should be measured within one or two days after beginning therapy.

Advice on fluid intake is helpful. Even patients who have done well on chronic thiazide therapy may still develop severe hyponatraemia if water intake increases. ${ }^{6}$ Patients need to know that diuretics will increase urine output, but that they should not subsequently seek deliberately to drink a lot: they only need drink as usual or when thirsty. Patients should be educated about the danger of drinking excessive water. Low doses of thiazide should be used in order to reduce the likelihood of development of TIH. ${ }^{3,6}$ In fact, only $10 \%$ of TIH cases occur at low dosage. ${ }^{3}$ Whether borderline hyponatraemia before initiation of treatment with a thiazide diuretic increases the likelihood of developing TIH is still unclear. However, low serum potassium is an independent risk factor for $\mathrm{TIH},{ }^{20}$ so it seems logical to propose appropriate potassium supplementation and combination with a potassium-sparing diuretic.

\section{Conclusions}

Hyponatraemia is a common complication of thiazide treatment. Careful monitoring of serum sodium, counselling about the risk of excessive fluid intake, and use of the lowest possible dosage will reduce the risk. Patients with severe and symptomatic hyponatraemia require urgent treatment with either saline or hypertonic saline with care to avoid excessively rapid restoration of serum sodium concentration. Patients with mild and asymptomatic hyponatraemia can usually be treated with withdrawal of the thiazide and restriction of fluid intake. 


\section{Acknowledgements}

Hull and East Yorkshire Hospitals, National Institute for Health Research (NIHR), Yorkshire and the Humber Deanery, and Hull York Medical School (HYMS).

\section{References}

1 Freis ED WA, Wilson IM, Parrish AE. Treatment of essential hypertension with chlorothiazide (diuril); its use alone and combined with other antihypertensive agents. J Am Med Assoc 1958;166:137-40.

2 Group. VAC-oS. Effects of treatment on morbidity in hypertension. Results in patients with diastolic blood pressures averaging 115 through 129 mm Hg. JAMA 1967;202:1028-34.

3 Mann SJ. The silent epidemic of thiazide-induced hyponatremia. J Clin Hypertens (Greenwich) 2008;10:477-84.

4 Fuisz RE, Lauler DP, Cohen P. Diuretic-induced hyponatremia and sustained antidiuresis. Am J Med 1962;33:783-91.

5 Gross P, Ketteler M, Hausmann C et al. Role of diuretics, hormonal derangements, and clinical setting of hyponatremia in medical patients. Klin Wochenschr 1988;66:662-9.

6 Spital A. Diuretic-induced hyponatremia. Am J Nephrol 1999;9:447-52.

7 Sonnenblick M, Friedlander Y, Rosin AJ. Diuretic-induced severe hyponatremia. Review and analysis of 129 reported patients. Chest 1993;103:601-6.

8 Chow KM, Szeto CC, Wong TY, Leung CB, Li PK. Risk factors for thiazide-induced hyponatraemia. QJM 2003;96:911-7.

9 Clark BA, Shannon RP, Rosa RM, Epstein FH. Increased susceptibility to thiazide-induced hyponatremia in the elderly. J Am Soc Nephrol 1994;5:1106-11.

10 Rose B. New approach to disturbances in the plasma sodium concentration. Am J Med 1986;81:1033-40.

11 Sloane E. Biology of women, 4th edn. London: Delmar, 2002:604-5.
12 Christensson T, Hellstrom K, Wengle B. Hypercalcemia and primary hyperparathyroidism. Prevalence in patients receiving thiazides as detected in a health screen. Arch Intern Med 1977;137:1138-42.

13 LaCroix AZ, Wienpahl J, White LR et al. Thiazide diuretic agents and the incidence of hip fracture. N Engl J Med 1990;322:286-90.

14 Ayus JC, Wheeler JM, Arieff AI. Postoperative hyponatremic encephalopathy in menstruant women. Ann Intern Med 1992;117:891-7.

15 Abramow MCE. Clinical aspects and pathophysiology of diureticinduced hyponatremia. Adv Nephrol Necker Hosp 1984;13:1-28.

16 Edelman IS LJ, O'Meara MP, Birkenfeld LW. Interrelations between serum sodium concentration, serum osmolality and total exchangeable sodium, total exchangeable potassium and total body water. J Clin Invest 1958;37:1236-56.

17 Chow KM KB, Szeto CC. Clinical studies of thiazide-induced hyponatremia. J Natl Med Assoc 2004;96:1305-8.

18 Adrogue $\mathrm{H}$. Consequences of inadequate management of hyponatremia. Am J Nephrol 2005;25:240-9.

19 Licata G, Di Pasquale P, Parrinello G et al. Effects of high-dose furosemide and small-volume hypertonic saline solution infusion in comparison with a high dose of furosemide as bolus in refractory congestive heart failure: long-term effects. Am Heart J 2003;145: $459-66$.

20 Byatt CM, Millard PH, Levin GE. Diuretics and electrolyte disturbances in 1000 consecutive geriatric admissions. J R Soc Med 1990;83:704-8.

Address for correspondence: Dr EEA Egom,

Castle Hill Hospital, University of Hull,

Cottingham, Kingston-upon-Hull, East Yorkshire HU16 5JQ.

Email: eeroumeaegom@doctors.org.uk

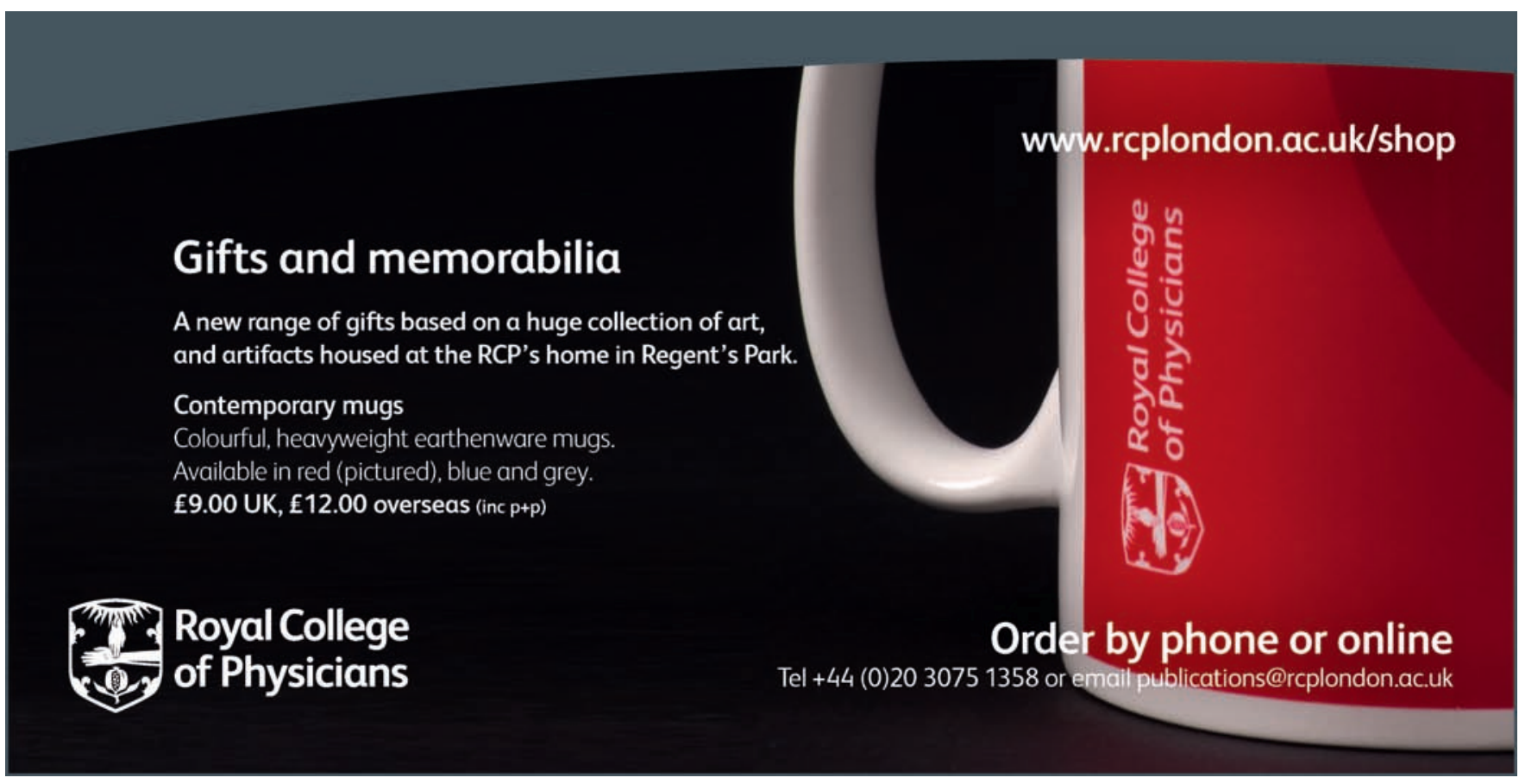

